\title{
Exercício aeróbio, de resistência ou combinado: que tipo mais contribui para melhorar a função endotelial? Resenha da metanálise de Ashor et al.
}

\section{Aerobic, Resistance or Combined Exercise: which Modality Most Contributes to Improving Endothelial Function? An Article View of The Meta-Analysis by Ashor et al.}

Felipe Keese Diogo Campos ${ }^{\S 1} \mathrm{MSc}$

Recebido em: 15 de dezembro de 2020. Aceito em: 22 de dezembro de 2020 Publicado online em: 29 de janeiro de 2021.

DOI: $10.37310 /$ ref.v89i4.2722

\begin{abstract}
Resumo
Introdução: Como parte da Edição Especial de 2020 I, a Revista de Educação Física / Journal of Physical Education (REF/JPE) está trazendo aspectos científicos da prática de atividade física relacionados à pandemia de CoViD-19.

Objetivo: Apresentar e indicar aos leitores da REF/JPE, a leitura do estudo de revisão, com metanálise, de Ashor et al., publicado em um periódico internacional. Baseado em estudos de ensaio clínico randomizados, os efeitos do exercício aeróbio, de resistência e combinado de ambos sobre a função endotelial foram comparados. A literatura recente indica que a CoViD-19 envolve o endotélio no processo, nesse sentido, avaliar qual o tipo de exercício melhora sua função é de alta relevância.

Conclusão: Todas as modalidades de exercício (aeróbio, resistidos e combinados) aumentaram a função endotelial significativamente em comparação com os grupos controle. Os maiores efeitos foram observados com os exercícios aeróbicos, seguidos pelos exercícios combinados e resistidos. Em exercícios aeróbios, houve uma relação dose-resposta significativa entre a intensidade tanto absoluta quanto relativa do exercício aeróbio e a função endotelial. Em exercícios resistidos a correlação positiva foi encontrada na frequência e não na intensidade. Os resultados foram discutidos pelos autores, recomenda-se a leitura.
\end{abstract}

Palavras-chave: exercício físico, endotélio, medicina preventiva.
Pontos Chave

- Todas as modalidades de exercício (aeróbio, resistidos e combinados) aumentaram a função endotelial.

- Os maiores efeitos foram observados com os exercícios aeróbicos, seguidos pelos exercícios combinados e resistidos.

- Exercícios aeróbios mostraram correlação positiva com melhora da função endotelial em intensidade. Enquanto em exercícios resistidos a correlação se deu na frequência.

\section{Abstract \\ Introduction: As part of the Special Edition 2020 I, the Revista de Educação Física / Journal of Physical Education (REF/JPE) brings scientific aspects of the practice of physical activity related to the CoViD-19 pandemic. \\ Objective: To present and refer to REF/JPE readers, the meta-analysis review study of Ashor et al,, published in an international scientific journal. Based on randomized clinical trial studies, the effects of aerobic, resistance and combined exercise on endothelial function were compared. Recent literature}

§Autor correspondente: Felipe Keese Diogo Campos - e-mail: felipecampos04@yahoo.com.br Afiliações: ${ }^{1}$ Instituto de Pesquisa da Capacitação Física do Exército, Rio de Janeiro-RJ, Brasil. 
indicates that CoViD-19 involves the endothelium in the process. In this sense, evaluating which type of exercise improves its function is highly relevant.

Conclusion: All types of exercise (aerobic, resistance and combined) significantly increased endothelial function compared to control groups. The greatest effects were observed with aerobic exercises, followed by combined and resisted exercises. In aerobic exercise, there was a significant doseresponse relationship between both absolute and relative intensity of aerobic exercise and endothelial function. In resistance exercises, a positive correlation was found in frequency and not in intensity. The results were discussed by the authors, reading is recommended

Keywords: exercise, endothelium, preventive medicine.

\author{
Key Points \\ - - All modalities of exercise \\ (aerobic, resistance and \\ combined) increased \\ endothelial function. \\ - The greatest effects were \\ observed with aerobic exercises, \\ followed by combined and \\ resisted exercises. \\ - Aerobic exercises showed a \\ positive correlation with \\ increasing in endothelial \\ function in intensity. While in \\ resistance exercises, the \\ correlation occurred in \\ frequency.
}

\section{Exercício aeróbio, de resistência ou combinado: que tipo mais contribui para melhorar a função endotelial? Resenha da metanálise de Ashor et al.}

\section{Introdução}

O início do ano de 2020 foi marcado pela ocorrência de uma pandemia de uma nova doença: a SARS-CoV2 (Severe Acute Respiratory Syndrome: síndrome respiratória aguda severa, causada por um novo coronavírus, o CoV2). A doença foi assim denominada porque, em 2002, o mundo já havia conhecido a pandemia causada por um coronavírus a SARS-CoV, que teve origem em Guandong, na China(1). Em dezembro de 2019, desta vez na província de Wuhan (China), registrouse o primeiro caso de infecção pelo Cov2(2). O vírus também causa SARS, porém, apresenta diversas outras características distintas, em comparação com o vírus anterior(3). Assim, a nova doença ficou conhecida como CoViD-19 (Corona Virus Disease from 2019).

Como parte da Edição Especial de 2020 parte I, a Revista de Educação Física / Journal of Physical Education (REF/JPE) está trazendo aspectos científicos da prática de atividade física relacionada à pandemia.
A literatura recente indica que a CoViD19 envolve o endotélio no processo, nesse sentido, avaliar qual o tipo de exercício melhora sua função tem sua relevância ressaltada.

O objetivo do presente trabalho foi apresentar e indicar aos leitores da $\mathrm{REF} / \mathrm{JPE}$, a leitura do estudo de revisão, com metanálise, de Ashor et al.(4), intitulado "Exercise modalities and endothelial function: a systematic review and dose-response meta-analysis of randomized controlled trials", publicado em um periódico internacional. Baseado em estudos de ensaio clínico randomizados, os efeitos do exercício aeróbio, de resistência e combinado de ambos sobre a função endotelial foram comparados.

\section{Desenvolvimento}

As doenças cardíacas isquêmicas representam a principal causa de morte no mundo, sendo responsável por cerca de 8,9 milhões de óbitos em 2019, de acordo com estimativas da Organização Mundial da Saúde(5). A disfunção endotelial é um 
importante preditor de doenças cardiovasculares, particularmente de cardiopatias isquêmicas(6) e está presente, também, no mecanismo patológico de acidente vascular cerebral(7), insuficiência renal crônica(8) e de uma variedade de doenças metabólicas, como obesidade, hipercolesterolemia e diabetes tipo II(9-12).

A disfunção endotelial é uma condição inflamatória, caracterizada por uma redução na biodisponibilidade de vasodilatadores deri-vados do endotélio, tais como o óxido nítrico, e um aumento, absoluto ou relativo, da biodisponibilidade de substâncias vasocons-tritoras. Este desequilíbrio prejudica a vasodilatação endotéliodependente, marca funcional que ilustra, portanto, a disfunção endotelial(13).

Do ponto de vista científico e epidemiológico, parece claro e consistente que a prática regular de exercícios físicos reduz signifi-cativamente a prevalência de doenças car-diovasculares(14-16), apresentando inclusive relação doseresposta inversa com doenças cardíacas isquêmicas(17). Uma das razões mais importantes é o fato de que o estresse de cisalhamento nos leitos vasculares, induzido pelo exercício físico, tanto de forma aguda quanto crônica, aumenta a síntese e liberação de óxido nítrico, melhorando a complacência vascular sistêmica e, consequentemente, a dinâmica circulatória(18-20).

Por outro lado, apesar de se reconhecer que a atividade física provoca efeitos muito positivos na saúde cardiovascular, os efeitos sobre marcadores de doenças cardiovasculares podem ser diferentes de acordo com a modalidade de exercício(21).

Dessa forma, Ashor et al.(4), por meio de uma revisão sistemática com meta-análise, buscaram investigar o efeito das modalidades de exercícios aeróbicos, resistidos e combinados na função endotelial. Em adição, procuraram determinar quais exercícios e características dos participantes são mais eficazes para melhorar a função endotelial.

Brevemente, o processo de seleção dos estudos a serem incluídos nessa revisão meta-analítica abrangeu somente: ensaios clínicos randomizados de exercícios com grupos com-parativos de controle (sem exercício); pro-tocolos de exercícios estruturados com duração igual ou superior a quatro semanas; indivíduos com idade igual ou superior a 18 anos; e estudos que mediram a função endotelial pelo método da dilatação fluxo-mediada da artéria braquial, em resposta a hiperemia reativa antes e após a intervenção experimental. O critério de análise da qualidade dos estudos selecionados foi avaliado a partir do escore de Jadad modificado (intervalo de 0 a 5), atribuindo dois pontos para uma randomização correta, dois pontos para métodos adequados de cegamento (cegamento da avaliação de resultados pelo operador) e um ponto para a descrição apropriada de abandono ou afastamento. Uma pontuação de Jadad inferior a três indicou estudo com baixa qualidade.

Assim, dos 785 estudos inicialmente encontrados, apenas 51 trabalhos foram considerados para a meta-análise, sendo dispostos em 65 ensaios independentes para fins de testagem dos efeitos de duas ou mais modalidades de exercícios na função endotelial.

\section{Conclusão}

Dentre os principais achados do estudo de Ashor et al.(4), destaca-se que todas as modalidades de exercício (aeróbio, resistidos e combinados) aumentaram a função endotelial significativamente em comparação com os grupos controle. Os maiores efeitos foram observados com os exercícios aeróbicos, seguidos pelos exercícios combinados e resistidos. Além disso, houve uma relação dose-resposta significativa entre a intensidade tanto absoluta quanto relativa do exercício aeróbio e a função endotelial. Em outras palavras, cada 2 MET de aumento na intensidade absoluta ou $10 \%$ de aumento na intensidade relativa $\left(\mathrm{VO}_{2}\right.$ pico $)$ foi associado a uma melhoria de $1 \%$ na função endotelial. Em contrapartida, em exercícios resistidos, a frequência, e não a intensidade, foi a variável positivamente associada com a função endotelial. Em relação aos exercícios combinados, foram observados 
efeitos favoráveis mais acentuados na função endotelial das pessoas com menores valores iniciais de dilatação fluxo-mediada. Tal informação pode indicar que exercícios resistidos e aeróbicos combinados em uma mesma sessão promovem ainda mais efeitos benéficos em indivíduos com maior risco cardiovascular.

A Figura 1 apresenta a página de face da citação(4). Confiram o importante e abrangente trabalho de Ashor et al.(4).

Springer Link
Systematic Review | Published: 04 October 2014
Exercise Modalities and Endothelial Function: A
Systematic Review and Dose-Response Meta-Analysis
of Randomized Controlled Trials
Ammar W. Ashor $⿴$, lose Lara, Mario Siervo Carlos Celis-Morales Clio Oggioni. Djordje G. Jakovjevvic \&
Sohn C. Mathers
2240 Accesses | 90 Citations | 21 Altmetric | Metrics
Abstract
Background
Regular exercise is associated with enhanced nitric oxide (NO) bioavailability. Flow-mediated
dilation (FMD) is used widely to assess endothelial function (EF) and NO release.
Objectives
The aims of this systematic review and meta-analysis were to (i) investigate the effect of
exercise modalities (aerobic, resistance or combined) on FMD; and (ii) determine which
exercise and oarticioant characteristics are most effective in imbroving. FMD.

Figura 1 - Ashor et al.(4) disponível em: doi:10.1007/s40279-014-0272-9

\section{Declaração de conflito de interesses}

Não há nenhum conflito de interesses em relação ao presente estudo.

\section{Declaração de financiamento}

Estudo conduzido sem financiamento.

\section{Referências}

1. Stadler $\mathrm{K}$, Masignani $\mathrm{V}$, Eickmann $\mathrm{M}$, Becker S, Abrignani S, Klenk H-D, et al. SARS--beginning to understand a new virus. Nature Reviews. Microbiology. [Online] 2003;1(3): 209-218. Available from: doi:10.1038/nrmicro775

2. Huang C, Wang Y, Li X, Ren L, Zhao J, Hu $\mathrm{Y}$, et al. Clinical features of patients infected with 2019 novel coronavirus in Wuhan, China. Lancet (London, England). [Online] 2020;395(10223): 497-506. Available from: doi:10.1016/S01406736(20)30183-5

3. Liu S-L, Saif L. Emerging Viruses without Borders: The Wuhan Coronavirus. Viruses.
[Online] 2020;12(2). Available from: doi:10.3390/v12020130

4. Ashor AW, Lara J, Siervo M, CelisMorales C, Oggioni C, Jakovljevic DG, et al. Exercise modalities and endothelial function: a systematic review and doseresponse meta-analysis of randomized controlled trials. Sports Medicine (Auckland, N.Z.). [Online] 2015;45(2): 279-296. Available from: doi:10.1007/s40279-014-0272-9

5. WHO. Global Health Estimates: Life expectancy and leading causes of death and disability. [Online] World Health Organization. Available from: https://www.who.int/data/maternalnewborn-child-adolescent/monitor [Accessed: 21st December 2020]

6. Widmer RJ, Lerman A. Endothelial dysfunction and cardiovascular disease. Global Cardiology Science \& Practice. [Online] 2014;2014(3): 291-308. Available from: doi: $10.5339 / \mathrm{gcsp} .2014 .43$

7. Cosentino F, Rubattu S, Savoia C, Venturelli V, Pagannonne E, Volpe M. Endothelial dysfunction and stroke. Journal of Cardiovascular Pharmacology. [Online] 2001;38 Suppl 2: S75-78. Available from: doi:10.1097/00005344200111002-00018

8. Martens CR, Kirkman DL, Edwards DG. The Vascular Endothelium in Chronic Kidney Disease: A Novel Target for Aerobic Exercise. Exercise and Sport Sciences Reviews. [Online] 2016;44(1): 12-19. Available from: doi:10.1249/JES.0000000000000065

9. Kawano H, Motoyama T, Hirai N, Kugiyama K, Yasue H, Ogawa H. Endothelial dysfunction in hypercholesterolemia is improved by Larginine administration: possible role of oxidative stress. Atherosclerosis. [Online] 2002;161(2): 375-380. Available from: doi:10.1016/s0021-9150(01)00671-2

10. Hartge MM, Unger T, Kintscher U. The endothelium and vascular inflammation in diabetes. Diabetes \& Vascular Disease Research. [Online] 2007;4(2): 84-88. Available from: doi: $10.3132 / \mathrm{dvdr} .2007 .025$

11. Meyers MR, Gokce N. Endothelial dysfunction in obesity: etiological role in 
atherosclerosis. Current Opinion in Endocrinology, Diabetes, and Obesity. [Online] 2007;14(5): 365-369. Available from:

doi:10.1097/MED.0b013e3282be90a8

12. Qiu S, Cai X, Yin H, Sun Z, Zügel M, Steinacker JM, et al. Exercise training and endothelial function in patients with type 2 diabetes: a meta-analysis. Cardiovascular Diabetology. [Online] 2018;17(1): 64. Available from: doi:10.1186/s12933-0180711-2

13. Storch AS, Mattos JD de, Alves R, Galdino I dos S, Rocha HNM, Storch AS, et al. Methods of Endothelial Function Assessment: Description and Applications. International Journal of Cardiovascular Sciences. [Online] Sociedade Brasileira de Cardiologia; 2017;30(3): 262-273. Available from: doi:10.5935/23594802.20170034

14. Laukkanen JA, Rauramaa R, Salonen JT, Kurl S. The predictive value of cardiorespiratory fitness combined with coronary risk evaluation and the risk of cardiovascular and all-cause death. Journal of Internal Medicine. [Online] 2007;262(2): 263-272. Available from: doi:10.1111/j.1365-2796.2007.01807.x

15. Löllgen H, Böckenhoff A, Knapp G. Physical activity and all-cause mortality: an updated meta-analysis with different intensity categories. International Journal of Sports Medicine. [Online] 2009;30(3): 213-224. Available from: doi:10.1055/s0028-1128150

16. Lear $\mathrm{SA}, \mathrm{Hu} \mathrm{W}$, Rangarajan $\mathrm{S}$, Gasevic D, Leong D, Iqbal R, et al. The effect of physical activity on mortality and cardiovascular disease in 130000 people from 17 high-income, middle-income, and low-income countries: the PURE study. Lancet (London, England). [Online] 2017;390(10113): 2643-2654. Available from: doi:10.1016/S0140-6736(17)316343

17. Kraus WE, Powell KE, Haskell WL, Janz KF, Campbell WW, Jakicic JM, et al. Physical Activity, All-Cause and Cardiovascular Mortality, and Cardiovascular Disease. Medicine and Science in Sports and Exercise. [Online] 2019;51(6): 1270-1281. Available from: doi:10.1249/MSS.0000000000001939
18. Green DJ, Maiorana A, O'Driscoll G, Taylor R. Effect of exercise training on endothelium-derived nitric oxide function in humans. The Journal of Physiology. [Online] 2004;561(Pt 1): 1-25. Available from: doi:10.1113/jphysiol.2004.068197

19. Gielen S, Schuler G, Adams V. Cardiovascular effects of exercise training: molecular mechanisms. Circulation. [Online] 2010;122(12): 1221-1238. Available from: doi:10.1161/CIRCULATIONAHA.110.93 9959

20. Schuler G, Adams V, Goto Y. Role of exercise in the prevention of cardiovascular disease: results, mechanisms, and new perspectives. European Heart Journal. [Online] 2013;34(24): 1790-1799. Available from: doi:10.1093/eurheartj/eht111

21. Pal S, Radavelli-Bagatini S, Ho S. Potential benefits of exercise on blood pressure and vascular function. Journal of the American Society of Hypertension. [Online] 2013;7(6): 494-506. Available from: doi:10.1016/j.jash.2013.07.004 